Quim. Nova, Vol. 35, No. 9, 1752-1757, 2012

\title{
ESTUDO TEÓRICO E EXPERIMENTAL DE ESPECTROS INFRAVERMELHO DE ÉSTERES DE ÁCIDO GRAXO PRESENTES NA COMPOSIÇÃO DO BIODIESEL DE SOJA
}

\author{
Eduardo W. Castilho-Almeida e Hélio F. Dos Santos* \\ Departamento de Química, Instituto de Ciências Exatas, Universidade Federal de Juiz de Fora, Campus Martelos, $36036-330$ Juiz \\ de Fora - MG, Brasil \\ Alisson Marques de Miranda e Ado Jorio \\ Departamento de Física, Instituto de Ciências Exatas, Universidade Federal de Minas Gerais, 30161-970 Belo Horizonte - MG, Brasil \\ Erlon H. Martins Ferreira, Carlos Alberto Achete e Raigna A. S. Z. Armond \\ Divisão de Materiais, Instituto Nacional de Metrologia, Normalização e Qualidade Industrial, Campus de Xerém, Av. Nossa Sra. \\ das Graças, 50, 25250-020 Duque de Caxias - RJ, Brasil \\ Cleber Paulo A. Anconi \\ Departamento de Química, Universidade Federal de Lavras, Campus Universitário, 37200-000 Lavras - MG, Brasil \\ Wagner Batista De Almeida \\ Departamento de Química, Instituto de Ciências Exatas, Universidade Federal de Minas Gerais, Av. Antônio Carlos, 6627, 31270- \\ 901 Belo Horizonte - MG, Brasil
}

Recebido em 20/12/11; aceito em 22/5/12; publicado na web em 24/8/12

\begin{abstract}
THEORETICAL AND EXPERIMENTAL STUDY OF INFRARED SPECTRA OF FATTY ACID ESTERS PRESENT IN SOYBEAN BIODIESEL. In this work, theoretical and experimental infrared spectra of fatty acid methyl esters (FAME) contained in soybean biodiesel were analyzed seeking the assignments of the relevant vibrational modes to characterize crude soybean oil and soybean biodiesel. The results showed the usefulness of infrared spectra for monitoring saturated and unsaturated compounds as well as impurities (mainly glycerol) in raw samples. This is the first step toward proposing an efficient molecular spectroscopy routine to certify biodiesel fuel.
\end{abstract}

Keywords: fatty acid esters; biodiesel; infrared spectra.

\section{INTRODUÇÃO}

No atual contexto mundial, o fator energético tem ganhado cada vez mais peso nas relações políticas, econômicas e sociais. As escassas reservas de petróleo ainda existentes no mundo anunciam uma catástrofe energética global em curto prazo. Além disso, os problemas ambientais inerentes ao uso de combustíveis de origem fóssil vêm, cada vez mais, contribuindo para a busca por fontes renováveis de energia. ${ }^{1,2}$ Neste cenário, diversas alternativas baseadas em biomassa têm se mostrado economicamente viáveis e ecologicamente sustentáveis. ${ }^{3-10}$ No Brasil, a matriz energética vem ganhando uma boa parcela de contribuição advinda de combustíveis de fontes renováveis. ${ }^{11}$ Para o biodiesel essa parcela é ainda pequena frente ao potencial de produção brasileiro (que se justifica pela grande disponibilidade de terras aptas ao cultivo de oleaginosas). Para efeito de comparação, no ano de 2010, a produção nacional de biodiesel puro (B100) foi de cerca de 2,4 bilhões de litros, aquém dos 2,6 bilhões de litros registrados pela Alemanha (país com uma área cultivável muito menor que a brasileira), líder mundial na produção deste combustível, no mesmo período. ${ }^{12-14}$

Uma das pretensões do governo brasileiro para acessar um mercado consumidor compatível com o potencial produtivo de biodiesel esperado é a exportação deste combustível para os países da União Europeia (UE). Todavia, esta estratégia depende de uma série de adequações de parâmetros físico-químicos do combustível, de acordo com determinações da Comissão Europeia (CE) para biocombustíveis, o que passa, necessariamente, por desenvolver parâmetros adequados para o controle de qualidade das fontes, bem como do produto final (biodiesel). No Brasil, uma Portaria de 2003 aliada a

*e-mail: helio.santos@ufjf.edu.br uma Resolução de 2004, ambas da Agência Nacional do Petróleo (ANP) ${ }^{15,16}$ trazem algumas especificações técnicas e metodológicas para a normatização da produção dos ésteres de ácidos graxos (EAG). Entretanto, diversas medidas ainda se fazem necessárias visando um melhor mapeamento do processo produtivo. Neste sentido, as análises relativas às composições dos óleos vegetais (triglicerídeos, TG) utilizados na síntese do referido biocombustível, bem como do composto base resultante (EAG) se traduzem em medidas auxiliares para a elucidação dos tipos de biodiesel obtidos com determinadas características físico-químicas, em função da composição química dos TG e EAG. A análise via espectroscopia vibracional surge neste ponto como uma boa ferramenta para este intento, haja vista o baixo custo da técnica, a reprodutibilidade e sensibilidade das análises. No presente trabalho, espectros infravermelho (IV) foram obtidos para ésteres metílicos de ácidos graxos (EMAG, constituintes básicos do biodiesel - Figura 1) e analisados visando a identificação de absorções características de tais sistemas.

Como técnica auxiliar, os espectros IV foram simulados utilizando níveis de teoria quânticos e confrontados com os dados experimentais. Os resultados serviram de base para estudos envolvendo a distinção de composições de biodiesel e a identificação de algumas impurezas prováveis no combustível (TG e glicerol).

\section{PARTE EXPERIMENTAL}

\section{Metodologia teórica}

As estruturas de EMAG trabalhadas foram propostas a partir da composição majoritária de TG no óleo de soja (Tabela 1). ${ }^{17,18}$ Tais moléculas foram submetidas inicialmente a uma dinâmica molecular 

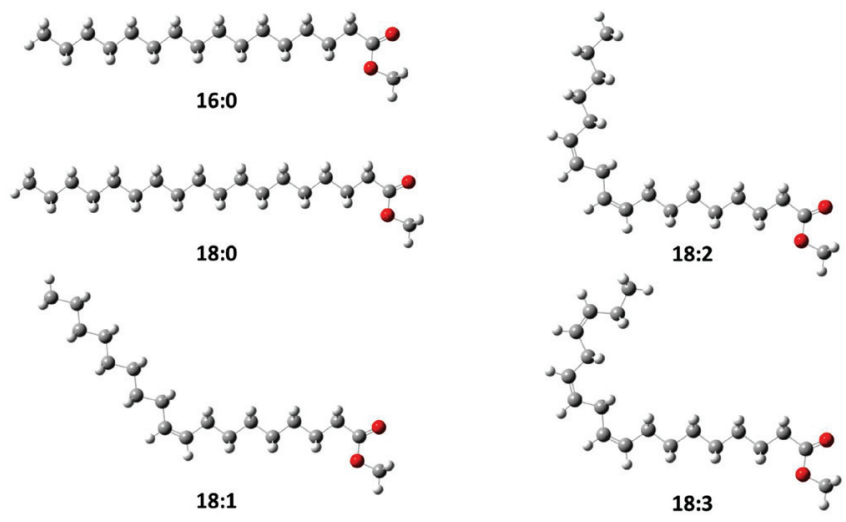

Figura 1. Estruturas dos EMAG trabalhados teórica e experimentalmente via análise espectroscópica na região do infravermelho

(DM) de 1.000 ps a $298,15 \mathrm{~K}$ utilizando o pacote computacional MacroModel ${ }^{\circledR 19}$ com o campo de força AMBER*, ${ }^{20,21}$ de acordo com um protocolo de aquecimento adequado para tal tipo de moléculas, ${ }^{22}$ visando um relaxamento da estrutura molecular. Neste processo, inúmeras estruturas são geradas ao longo da trajetória, fazendo com que uma seleção seja necessária visando a obtenção de uma estrutura representativa do sistema, a qual será utilizada em cálculos quânticos sequenciais. Neste sentido, as estruturas finais do processo de DM que apresentaram valores de energia próximos à média para o processo de simulação são preferenciais para a continuação da análise. Em termos estruturais, pode-se dizer que nesta situação encontramos geometrias com correspondência estrutural, fato que se reflete pela análise do desvio padrão médio quadrático (Root Mean Square Displacement, RMSD) em relação à estrutura inicial; após um determinado tempo, as estruturas convergem para um padrão de estabilidade que possibilita a escolha das mesmas para a próxima etapa do protocolo de modelagem (Figura 2).

As estruturas selecionadas através da DM foram então otimizadas no nível de teoria SVWN²3/6-31G como implementado no pacote de métodos computacionais Gaussian $03^{\circledR} \cdot{ }^{24}$ Posteriormente, foram realizados cálculos de frequências harmônicas e intensidades vibracionais utilizando o mesmo nível de teoria aplicado para a otimização das estruturas.

Os espectros IV teóricos foram obtidos para todos os compostos apresentados na Figura 1. As representações relativas às cadeias dos EMAG são dadas pelo número de carbonos da cadeia seguido do número de insaturações da mesma (por exemplo, 18:2 - cadeia de dezoito carbonos com duas insaturações). Os espectros de bandas teóricos foram obtidos através do ajuste das intensidades e números de onda calculados numa soma de funções do tipo Lorentziana (Equação $1)^{25}$ utilizando os parâmetros de largura da banda à meia altura $\left(\omega_{i}\right) \mathrm{e}$ concentração $(n)$ iguais a, respectivamente, $15 \mathrm{~cm}^{-1} \mathrm{e} 1 \times 10^{-5} \mathrm{~mol} \mathrm{~cm} \mathrm{~cm}^{-3}$.

$$
A(v)=\left[\ln \left(\frac{I_{0}}{I}\right)\right]=n l \sum_{i=1}^{3 n-6}\left[A_{i} \frac{2 \omega_{i}}{\pi} \frac{1}{4\left(v-v_{i}\right)^{2}+\omega_{i}^{2}}\right]
$$

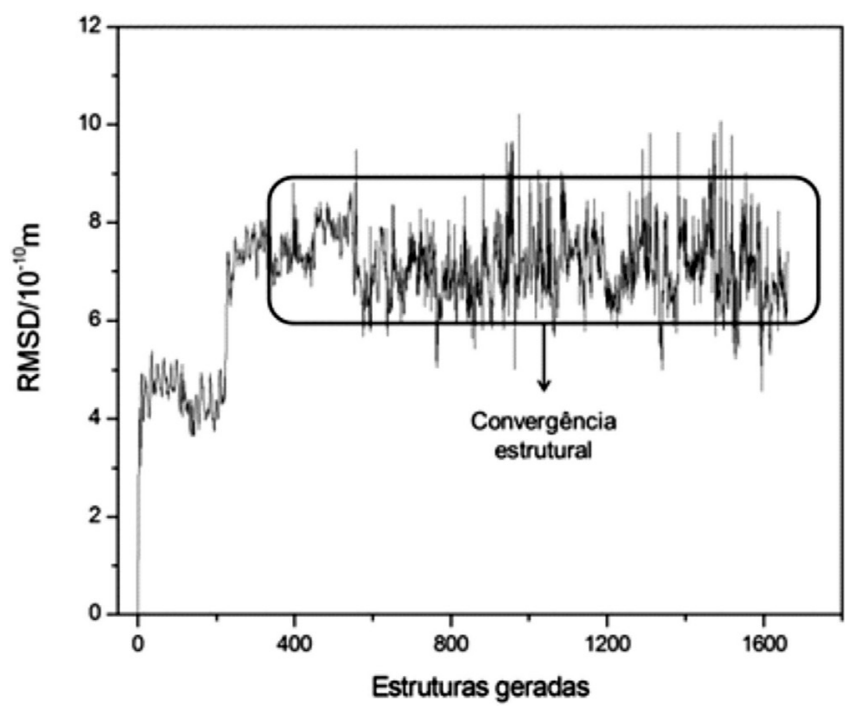

Figura 2. Análise de RMSD para a trajetória da DM do EMAG 18:3 evidenciando a convergência estrutural

Nesta equação, a absorbância ajustada, $A(v)$ se relaciona com a concentração da amostra, $n\left(\mathrm{~mol} \mathrm{~cm}{ }^{-3}\right)$; o caminho óptico, $l(\mathrm{~cm})$; a intensidade do modo de vibração, $A_{i}\left(\mathrm{~cm} \mathrm{~mol}^{-1}\right)$ e sua respectiva frequência $v_{i}\left(\mathrm{~cm}^{-1}\right)$ e a largura da banda à meia altura, $\omega_{i}$. Esses parâmetros contribuem para o ajuste do perfil espectral. Os únicos parâmetros a serem inseridos para a elaboração do ajuste são $n, l, \omega_{i}$ e a região espectral a ser contemplada.

Devido à aproximação harmônica (mecânica e elétrica) utilizada para o cálculo das frequências e intensidades vibracionais, os resultados teóricos apresentam desvios em relação aos valores experimentais, os quais são dependentes do nível de teoria empregado. De acordo com Fan e Ziegler, ${ }^{26}$ frequências vibracionais são menos sensíveis ao tipo de funcional (local ou não local) e função de base, entretanto, para previsão de intensidades, correções não local para o funcional e funções de base de qualidade superior a duplo zeta são necessárias. Portanto, considerando que o nível de teoria empregado aqui (SVWN/6-31G) utiliza uma aproximação local para o funcional e uma função de base modesta (duplo zeta de valência não polarizada) são esperados desvios no perfil espectral. Com o objetivo de minimizar as diferenças entre teoria e experimento, possibilitando uma análise mais quantitativa, as frequências vibracionais calculadas $\left(v_{i}^{\text {calc }}\right)$ foram escalonadas, anteriormente à aplicação da Equação 1, utilizando o procedimento padrão, ou seja, $\tilde{v}_{i}^{\text {scal. }}=\lambda \tilde{v}_{i}^{\text {calc. }}$, sendo $\lambda \mathrm{o}$ fator de escala determinado de acordo com a Equação 2:27,28

$$
\lambda=\sum_{i}^{n} \tilde{v}_{i}^{\text {calc. }} \tilde{v}_{i}^{\text {expt. }} / \sum_{i}^{n}\left(\tilde{v}_{i}^{\text {calc. }}\right)^{2}
$$

Neste estudo, diferentes valores de $\lambda$ foram obtidos para cada molécula estudada em três regiões distintas, i.e., 3500-2200, 22001600 e 1600-600 $\mathrm{cm}^{-1}$. Os resultados são mostrados na Tabela 2. Para as intensidades não há um procedimento padrão para escalonamento,

Tabela 1. Composição dos derivados de ácidos graxos em algumas fontes de óleos ${ }^{17,18}$

\begin{tabular}{lcccccc}
\hline Óleo vegetal & Palmítico (16:0) & Esteárico (18:0) & Palmitoleico (16:1) & Oleico (18:1) & Linoleico (18:2) & Outros \\
\hline Óleo de oliva & 14,6 & - & - & 75,4 & 10,0 & - \\
Óleo de girassol & 6,4 & 2,9 & 0,1 & 17,7 & 72,8 & 0,1 \\
Óleo de algodão & 28,6 & 0,9 & 0,1 & 13,0 & 47,2 & 0,2 \\
Óleo de milho & 6,0 & 2,0 & - & 20,0 & 48,0 & 64,0 \\
Óleo de soja & 11,0 & 2,0 & - & - & 3,0 \\
\hline
\end{tabular}


Tabela 2. Fatores de escalonamento $(\lambda)$ derivados no presente trabalho para correção de frequências vibracionais de EMAG calculadas no nível SVWN/6-31G

\begin{tabular}{ccccccc}
\hline \multirow{2}{*}{ Regiões } & \multicolumn{5}{c}{ EMAG } \\
\cline { 2 - 7 } & $16: 0$ & $18: 0$ & $18: 1$ & $18: 2$ & $18: 3$ & 0,96929 \\
\hline $3500-2200 \mathrm{~cm}^{-1}$ & 0,96556 & 0,96383 & 0,97091 & 0,96968 & $1,968 \pm 0,003$ \\
$2200-1600 \mathrm{~cm}^{-1}$ & 1,0040 & 1,0122 & 1,0099 & 1,0034 & 1,0122 & $1,008 \pm 0,004$ \\
$1600-600 \mathrm{~cm}^{-1}$ & 0,99648 & 0,99842 & 1,0014 & 1,0022 & 0,99466 & $0,999 \pm 0,003$ \\
\hline
\end{tabular}

devido à alta sensibilidade dessa propriedade com o nível e teoria, portanto, os valores originais para $A_{i}\left(\mathrm{em} \mathrm{cm} \mathrm{mol}^{-1}\right)$ foram empregados na Equação 1.

\section{Metodologia experimental}

Os espectros experimentais foram obtidos em um espectrômetro infravermelho por transformada de Fourier, da Perkin-Elmer (FT-IR System Spectrum GX), localizado na Divisão de Materiais (DIMAT) do INMETRO. Os espectros relativos aos triglicerídeos e ésteres de ácido graxo foram coletados na região do infravermelho médio (400-4000 cm-1) em uma sequência de 10 scans com resolução de $4,00 \mathrm{~cm}^{-1}$. Nas medidas experimentais foi utilizada uma célula para ATR plana equipada com uma placa plana de ZnSe. Antes de cada medida, a referida célula foi limpa com álcool etílico e submetida à medida enquanto vazia, visando a obtenção do espectro branco com a eliminação do $\mathrm{CO}_{2}$ e $\mathrm{H}_{2} \mathrm{O}$.

$\mathrm{Na}$ análise efetuada foram utilizados ésteres metílicos de ácido graxo de alta pureza $(99+\mathrm{m} / \mathrm{m} \%$ - Sigma-Aldrich). Os ésteres puros permaneceram conservados sob refrigeração até o momento da medida. Dentre os 5 EMAG trabalhados, 3 apresentam-se no estado líquido (18:1, 18:2 e 18:3) e os outros 2 no estado sólido (16:0 e 18:0). Nos casos de espectros envolvendo misturas de ésteres, estas foram confeccionadas a partir da pesagem dos referidos compostos nas proporções molares estabelecidas, sendo a mistura submetida a banho de ultrassom por 30 min em frasco de vidro âmbar, visando a homogeneização do sistema.

\section{RESULTADOS E DISCUSSÃO}

\section{Análise dos espectros de EMAG puros}

Os espectros IV teóricos podem ser utilizados como dados auxiliares para a atribuição de espectros experimentais. $\mathrm{Na}$ análise computacional, todos os $3 \mathrm{~N}-6$ modos vibracionais fundamentais (onde $\mathrm{N}$ equivale ao número de átomos contidos no sistema molecular) associados à estrutura molecular se tornam acessíveis de maneira independente, o que permite a distinção dos modos constituintes das principais bandas observadas experimentalmente. ${ }^{29}$ Neste sentido, torna-se importante a comparação direta dos perfis espectrais obtidos pelas duas técnicas, principalmente no que tange à frequência determinada para os modos vibracionais. Os espectros IV para EMAG puros são representados nas Figuras 3 (experimentais) e 4 (teóricos). Como pode ser prontamente observado, o número de bandas previstas na teoria é menor em função do uso de uma única molécula para o cálculo do espectro IV, ou seja, não há conformações distintas ou efeitos de matriz (interações intermoleculares) incluídos na modelagem dos sistemas. Entretanto, o perfil espectral com bandas intensas concentradas em regiões específicas é satisfatoriamente reproduzido.

Os espectros foram divididos em quatro regiões para facilitar a análise, quais sejam: R1 (2500-3500 cm-1), R2 (1500-2000 cm-1), R3 $\left(1000-1500 \mathrm{~cm}^{-1}\right)$ e R4 $\left(<1000 \mathrm{~cm}^{-1}\right)$. Em R1, pode-se notar a existência de três bandas claramente identificáveis. Estas bandas constituem-se da junção de vários modos vibracionais relacionados a porções distintas

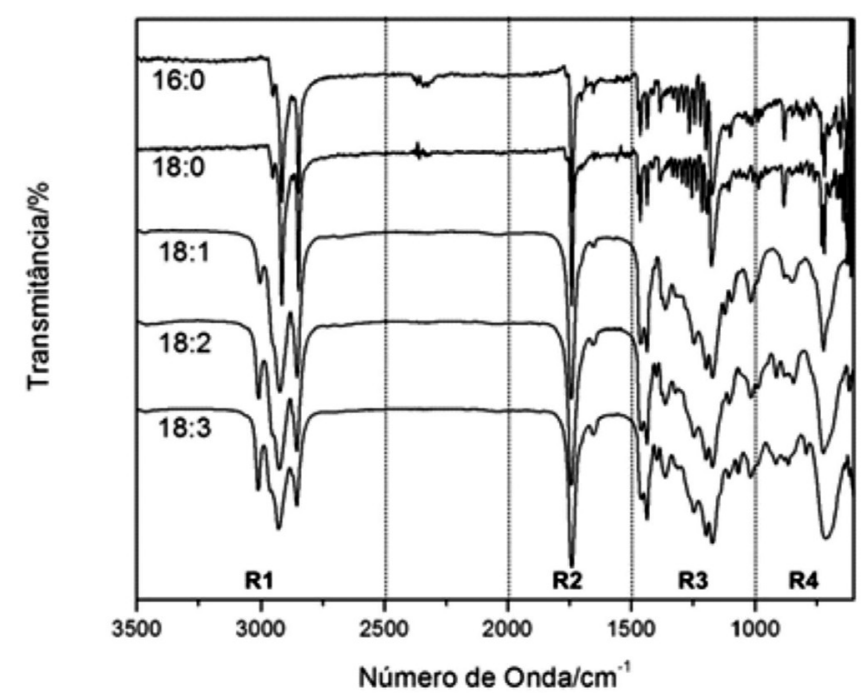

Figura 3. Espectros infravermelho experimentais de cinco ésteres metílicos de ácidos graxos presentes no biodiesel derivado do óleo de soja $\left(16: 0_{(s)}, 18: 0_{(s)}\right.$, $18: 1_{(l)}, 18: 2_{(l)}$ e 18:3 $(l)$ evidenciando as diferenças relativas ao aumento do número de insaturações

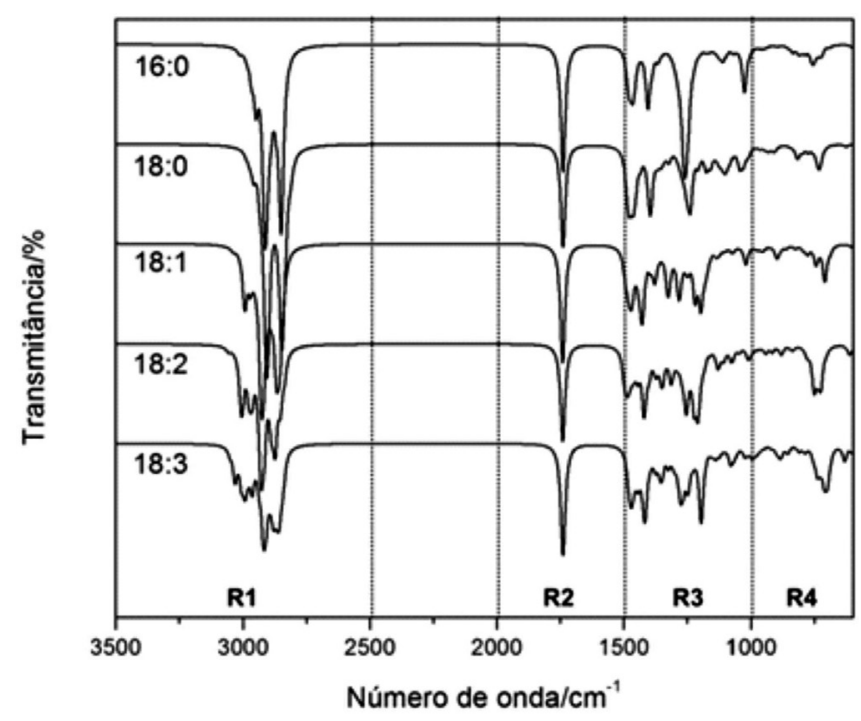

Figura 4. Espectros infravermelho teóricos dos EMAG estudados (16:0, 18:0, 18:1, 18:2 e 18:3). Os fatores de escalonamento utilizados para as frequências vibracionais são mostrados na Tabela 2

da molécula, dentre os quais se destacam os modos de estiramento simétrico e assimétrico para a ligação $\mathrm{C}-\mathrm{H}$ de grupos metílicos $\left(\mathrm{v}_{\mathrm{s}} \mathrm{C}-\mathrm{H}\right.$ e $\left.\mathrm{V}_{\mathrm{as}} \mathrm{C}-\mathrm{H}\right)\left(2850-2960 \mathrm{~cm}^{-1}\right)$ e estiramento simétrico e assimétrico das ligações $\mathrm{C}-\mathrm{H}$ em grupos olefínicos $\left(\mathrm{v}_{\mathrm{s}} \mathrm{C}-\mathrm{H}_{\text {olef }}\right.$ e $\left.\mathrm{v}_{\mathrm{as}} \mathrm{C}-\mathrm{H}_{\text {olef }}\right)(3000-3015$ $\mathrm{cm}^{-1}$ ) para os derivados insaturados (18:1, 18:2 e 18:3).

A banda com frequência mais alta próxima a $3000 \mathrm{~cm}^{-1}\left(v_{\mathrm{s}} \mathrm{C}-\mathrm{H}_{\text {olef }}\right.$ e $v_{\text {as }} \mathrm{C}-\mathrm{H}_{\text {olef }}$ ) sofre um pequeno aumento de intensidade, proporcional ao número de insaturações presentes na molécula do éster metílico 
analisado. Este comportamento pode ser verificado nas Figuras 3 e 4, portanto, percebe-se que existe uma identidade espectral atrelada à presença de insaturações na amostra a ser analisada. Tal constatação possui grande apelo, uma vez que muitos dos parâmetros físico-químicos de análise de qualidade de biodiesel passam pela quantificação do percentual de compostos insaturados na composição do referido combustível.

A análise da segunda região espectral (R2, 1500-2000 $\left.\mathrm{cm}^{-1}\right)$ mostra uma banda intensa em $1740 \mathrm{~cm}^{-1}$ atribuída ao estiramento da ligação $\mathrm{C}=\mathrm{O}$ de ésteres $(\mathrm{v}(\mathrm{C}=\mathrm{O})$. Para os derivados insaturados é também observada uma banda de absorção próxima a $1650 \mathrm{~cm}^{-1}$, relativa ao estiramento da ligação dupla $\mathrm{C}=\mathrm{C}(\mathrm{v}(\mathrm{C}=\mathrm{C}))$. Nos espectros teóricos de EMAG (Figura 4), estes dois modos são degenerados dando origem a uma única banda centrada em $1740 \mathrm{~cm}^{-1}$ (a intensidade relativa da banda $v(C=C)$ em relação à $v(C=O)$ para o derivado 18:1 é 0,024:1). Nesta região, a presença da banda em $1650 \mathrm{~cm}^{-1}$ pode, portanto, ser utilizada como sonda para identificação de EMAG insaturados.

Em R3 (1000-1500 $\mathrm{cm}^{-1}$ ), o perfil do espectro é complexo e relativamente sensível para os EMAG considerados. Para todos os compostos trabalhados, são observadas duas bandas muito próximas e de média intensidade entre $1430-1480 \mathrm{~cm}^{-1}$, que se relacionam aos modos de deformação angular simétrica no plano para $\mathrm{CH}_{2}-\delta_{\mathrm{s}}\left(\mathrm{CH}_{2}\right)$ - e deformação assimétrica de $\mathrm{CH}_{3}-\delta_{\mathrm{as}}\left(\mathrm{CH}_{3}\right)$. Nota-se que para os ésteres insaturados essas bandas são mais sobrepostas, devido à contribuição do modo $\delta_{\text {as }}\left(\mathrm{C}-\mathrm{H}\right.$ olefínico) próximo a $1430 \mathrm{~cm}^{-1}$. Para os ésteres insaturados, é observada outra absorção característica em $\mathrm{R} 3$ próxima a $1280 \mathrm{~cm}^{-1}$ atribuída ao modo $\delta_{s}(\mathrm{C}-\mathrm{H}$ olefínico), o qual possui intensidade semelhante ao modo assimétrico correspondente. As bandas que aparecem abaixo de $1250 \mathrm{~cm}^{-1}$ se apresentam associadas às deformações angulares assimétricas e simétricas fora do plano para $\mathrm{CH}_{2}$ $-\tau\left(\mathrm{CH}_{2}\right)$ e $\omega\left(\mathrm{CH}_{2}\right)$, respectivamente -, com intensidades medianas, e ao estiramento assimétrico associado ao grupamento funcional de ésteres $\left(\mathrm{v}_{\mathrm{as}}\left[\mathrm{CH}_{2}(\mathrm{C}=\mathrm{O}) \mathrm{OCH}_{3}\right]\right)$, os quais apresentam intensidades moderadas. Outra banda que merece atenção é a localizada próxima a 1000-1090 $\mathrm{cm}^{-1}$, relativa ao estiramento da ligação C-O não acoplada de ésteres.

Na região R4, de baixa frequência, os modos mais importantes são atribuídos às deformações angulares fora do plano dos grupamentos $\mathrm{C}=\mathrm{C}-\mathrm{H}-\tau(\mathrm{C}-\mathrm{H}$ olefínico $)$ e $\omega(\mathrm{C}-\mathrm{H}$ olefínico $)$ para os derivados insaturados. Estas frequências características calculadas para os derivados insaturados apresentam-se entre $1000-700 \mathrm{~cm}^{-1}$, com uma banda intensa próxima a $720 \mathrm{~cm}^{-1}$. Note que a intensidade calculada para esse modo é subestimada devido, principalmente, à limitação do método teórico utilizado (ver ref. 26), no entanto, o padrão observado para EMAG saturados e insaturados é mantido, sendo as bandas mais intensas para EMAG insaturados. A banda fina e pouco intensa verificada para os derivados saturados (e também nos insaturados, porém sobreposta) deve-se ao modo de deformação angular assimétrica no plano $\left(\rho\left(\mathrm{CH}_{2}\right) \sim 730 \mathrm{~cm}^{-1}\right)$. Os comentários efetuados anteriormente apresentam-se de maneira organizada na Tabela 3 referente aos EMAG.

As conclusões obtidas através da análise dos espectros teóricos e experimentais dos EMAG puros permitem uma distinção entre o perfil espectral de compostos saturados e insaturados, em virtude da presença de bandas características de derivados com estas naturezas químicas. Especificamente, essas bandas aparecem em regiões distintas do espectro, ou seja, próximo a $3000 \mathrm{~cm}^{-1}$ em R1 $\left(v_{\mathrm{s}} \mathrm{C}-\mathrm{H}_{\text {olef }}\right.$ e $\mathrm{v}_{\text {as }} \mathrm{C}-$ $\left.\mathrm{H}_{\text {olef }}\right), 1650 \mathrm{~cm}^{-1}$ em R2 ( $\left.\mathrm{vC}=\mathrm{C}\right), 1420-1430 \mathrm{~cm}^{-1}$ em R3 $\left(\delta_{\text {as }} \mathrm{C}-\mathrm{H}_{\text {olef }}\right)$ e próximo a $720 \mathrm{~cm}^{-1} \omega(\mathrm{C}-\mathrm{H}$ olefínico). Para evidenciar tais diferenças em matrizes que mimetizam o biodiesel, foram efetuadas medidas de IV experimentais para misturas de EMAG em proporções conhecidas, concomitantemente à simulação de alguns espectros teóricos de EMAG a partir dos principais constituintes do óleo de soja (Tabela 1).

\section{Análise dos espectros de misturas de EMAG}

As misturas experimentais foram efetuadas de modo simples, através da mescla de quantidades determinadas dos padrões de EMAG. Os ajustes dos espectros simulados foram efetuados de forma aproximada, conforme a Equação 3; as transmitâncias $\left(T_{i}\right)$ obtidas nos cálculos dos espectros IV teóricos de cada componente $(i)$ do biodiesel foram multiplicadas por uma fração molar $\left(\chi_{i}\right)$ correspondente obtida da literatura. ${ }^{30}$ Tais transmitâncias ponderadas são então somadas, resultando na transmitância final da mistura $\left(T_{t}\right)$.

$$
T_{t}=\sum_{i}^{n} \chi_{i} \cdot T_{i}
$$

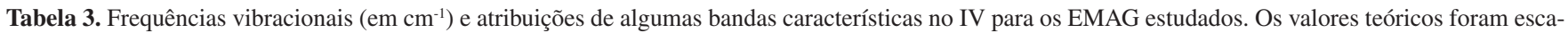
lonados de acordo com a Tabela 2

\begin{tabular}{|c|c|c|c|c|c|c|c|c|c|c|c|}
\hline & \multirow{2}{*}{ Atribuição } & \multicolumn{2}{|c|}{$(16: 0)$} & \multicolumn{2}{|c|}{$(18: 0)$} & \multicolumn{2}{|c|}{$(18: 1)$} & \multicolumn{2}{|c|}{$(18: 2)$} & \multicolumn{2}{|c|}{$(18: 3)$} \\
\hline & & Teórico & Exp. & Teórico & Exp. & Teórico & Exp. & Teórico & Exp. & Teórico & Exp. \\
\hline \multirow{5}{*}{ R1 } & $v_{s}(\mathrm{C}-\mathrm{H})_{\text {olef }}$ & - & - & - & - & 2993 & 3004 & 3006 & 3009 & 3032 & 3011 \\
\hline & $v_{\text {as }}(\mathrm{C}-\mathrm{H})_{\text {olef }}$ & - & - & - & - & 2974 & 3004 & 2970 & 3009 & 2965 & 3011 \\
\hline & $\mathrm{v}_{\mathrm{as}}\left(\mathrm{CH}_{3}\right)$ & 2949 & 2952 & 2958 & 2952 & 2974 & 2948 & 2970 & 2951 & 2965 & 2961 \\
\hline & $v_{\text {as }}(\mathrm{C}-\mathrm{H})$ & 2914 & 2915 & 2907 & 2916 & 2926 & 2923 & 2928 & 2926 & 2919 & 2928 \\
\hline & $v_{s}(\mathrm{C}-\mathrm{H})$ & 2851 & 2847 & 2851 & 2848 & 2866 & 2854 & 2876 & 2855 & 2886 & 2855 \\
\hline \multirow{2}{*}{ R2 } & $v(\mathrm{C}=\mathrm{O}$ éster $)$ & 1740 & 1740 & 1740 & 1740 & 1741 & 1741 & 1741 & 1741 & 1740 & 1740 \\
\hline & $v(C=C)$ & & - & & - & 1741 & 1648 & 1741 & 1656 & 1740 & 1652 \\
\hline \multirow{7}{*}{ R3 } & $\delta_{\mathrm{as}}\left(\mathrm{CH}_{3}\right) / \delta_{\mathrm{s}}\left(\mathrm{CH}_{2}\right)$ & 1466 & 1463 & 1478 & 1463 & 1472 & 1463 & 1485 & 1457 & 1482 & 1456 \\
\hline & $\delta_{\mathrm{s}}\left(\mathrm{CH}_{3}\right)$ & 1405 & 1435 & 1396 & 1435 & 1429 & 1435 & 1420 & 1435 & 1442 & 1435 \\
\hline & $\delta_{\mathrm{as}}(\mathrm{C}-\mathrm{H}$ olefínico $)$ & & - & & - & 1429 & 1421 & 1420 & 1419 & 1427 & 1419 \\
\hline & $\delta_{\mathrm{s}}(\mathrm{C}-\mathrm{H}$ olefínico $)$ & & - & & - & 1283 & 1279 & 1254 & 1278 & 1284 & 1277 \\
\hline & $\omega\left(\mathrm{CH}_{2}\right)$ & 1262 & 1264 & 1247 & 1254 & 1246 & 1246 & 1224 & 1245 & 1230 & 1245 \\
\hline & $v_{\text {as }}\left[\mathrm{CH}_{2}(\mathrm{C}=\mathrm{O}) \mathrm{OCH}_{2}\right] / \tau\left(\mathrm{CH}_{2}\right)$ & 1261 & $1309 / 1172$ & 1240 & $1313 / 1175$ & 1197 & 1196 & 1210 & 1196 & 1205 & 1196 \\
\hline & $v(\mathrm{C}-\mathrm{O}$ éster $)$ & 1027 & 1015 & 1045 & 1009 & 1021 & 1017 & 1011 & 1016 & 1004 & 1018 \\
\hline \multirow{3}{*}{ R4 } & $\tau(\mathrm{C}-\mathrm{H}$ olefínico $)$ & - & - & - & - & 976 & 994 & 971 & 988 & 1004 & 994 \\
\hline & $\omega(\mathrm{C}-\mathrm{H}$ olefínico $)$ & - & - & - & - & 710 & 723 & 740 & 723 & 730 & 711 \\
\hline & $\rho\left(\mathrm{CH}_{2}\right)$ & 757 & 730 & 732 & 730 & 745 & 756 & 744 & 754 & 738 & 719 \\
\hline
\end{tabular}


Os espectros obtidos são representados na Figura 5 para misturas distintas contendo EMAG saturados e insaturados em igual quantidade $(50 \% 16: 0+50 \%$ 18:2) e contendo uma maior proporção molar de derivados insaturados ( $15 \%$ 16:0 + 85\% 18:2). As diferenças entre as duas misturas são sutis, entretanto, as bandas marcadoras indicadas para os EMAG puros podem ser identificadas e utilizadas como características das misturas. Na primeira região, R1, a absorção próxima a $3000 \mathrm{~cm}^{-1}$ é característica de ésteres insaturados e, portanto, apresenta maior intensidade na mistura rica em 18:2. Como esta banda é exclusiva de derivados insaturados, serve de marcador qualitativo, $a$ priori, da relação percentual entre compostos insaturados e saturados em uma mistura de biodiesel comercial.

Na região R2 observa-se claramente a intensificação da banda em $1650 \mathrm{~cm}^{-1}$ na mistura contendo maior quantidade de $18: 2$, característica de EMAG insaturados. Como discutido anteriormente, os modos $v(C=C)$ e $v(C=O)$ são calculados com a mesma frequência, como representado na Figura $5 \mathrm{~b}$ pela banda centrada em $1740 \mathrm{~cm}^{-1}$. Analisando a região R3, as duas misturas apresentam perfis muito semelhantes e similares àqueles observados para os EMAG insaturados (Figura 3). As principais bandas que definem o perfil da região R3 são observadas na faixa $1100-1250 \mathrm{~cm}^{-1}$. Próximo a $1400 \mathrm{~cm}^{-1}$
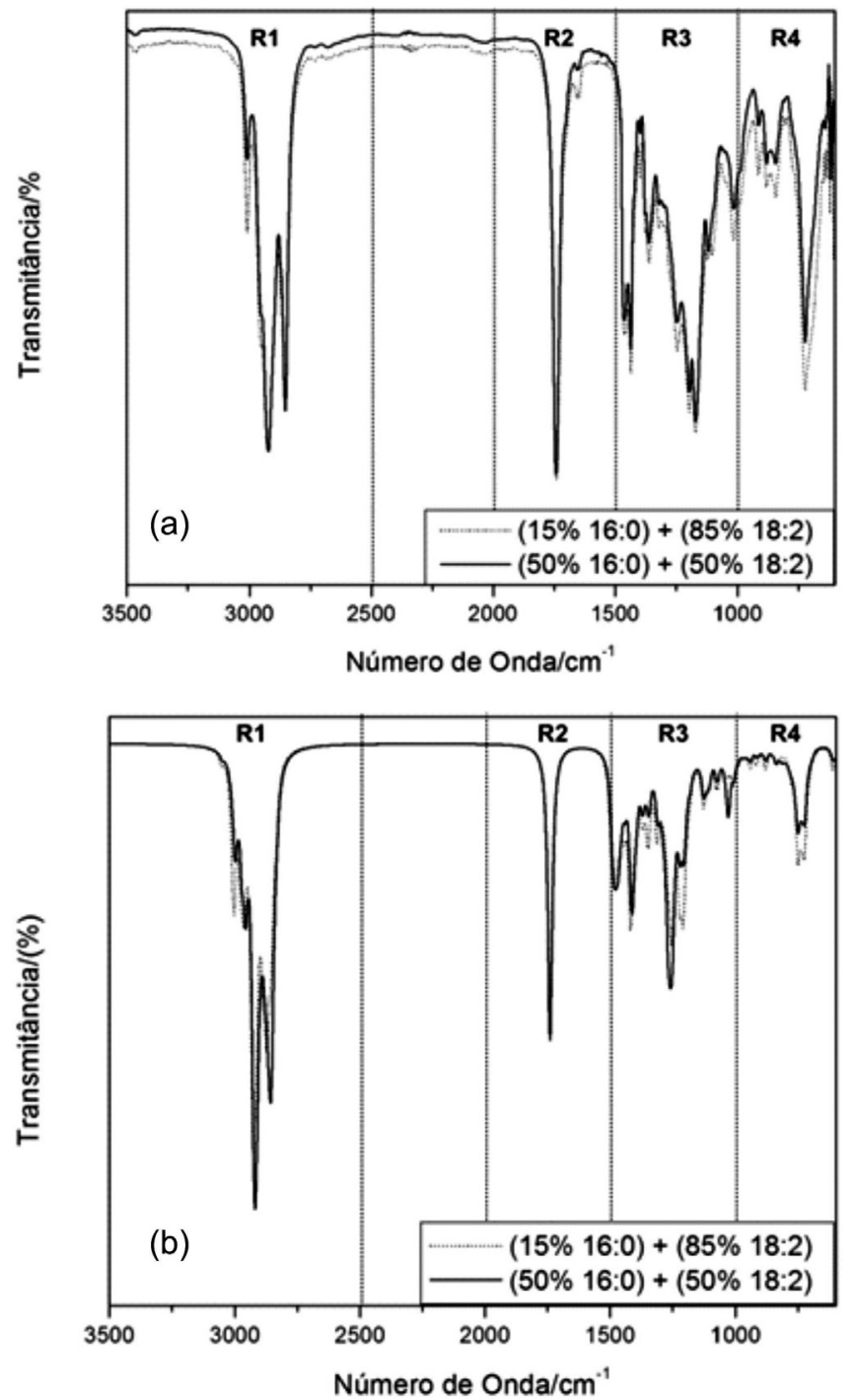

Figura 5. Comparação entre os espectros infravermelho experimental (a) e teórico (b) das misturas contendo $15 \%$ de 16:0 + 85\% de 18:2 e 50\% de $16: 0+85 \%$ de 18:2 evidenciando as diferenças relativas ao percentual de derivados insaturados observa-se uma banda com intensidade distinta no comparativo entre as misturas rica e pobre em compostos insaturados. No caso, o modo de deformação angular assimétrica no plano em $\mathrm{C}=\mathrm{C}-\mathrm{H}-\delta_{\text {as }}(\mathrm{C}-\mathrm{H}$ olefínico) - previsto teoricamente indica a distinção na concentração de derivados insaturados nas duas misturas consideradas. Finalmente, na região $\mathrm{R} 4$, próximo a $1000 \mathrm{~cm}^{-1}$, são notadas três bandas que se associam aos modos de estiramento $\mathrm{C}-\mathrm{O}$ de ésteres $-\mathrm{v}(\mathrm{C}-\mathrm{O}$ éster $)$ - ao estiramento assimétrico alifático $\mathrm{CH}_{2}-\mathrm{CH}_{2}-\mathrm{CH}_{2}-\mathrm{v}_{\mathrm{as}}(\mathrm{CCC})$ e à deformação angular assimétrica de $\mathrm{C}-\mathrm{H}$ olefínico fora do plano $\tau(\mathrm{C}-\mathrm{H}$ olefínico). Estes últimos modos aparecem muito próximos em duas bandas geminadas. As diferenças verificadas entre as misturas se devem, como esperado, à diferença no percentual de insaturações nos dois casos, fato este que se refere diretamente ao último modo citado. Ainda em R4, a maior intensidade da banda próxima a $720 \mathrm{~cm}^{-1}$ é indicativa da presença de EMAG insaturados, em função do modo vibracional de deformação angular simétrica de $\mathrm{C}=\mathrm{C}-\mathrm{H}$ olefínico fora do plano $-\omega(\mathrm{C}-\mathrm{H}$ olefínico $)$.

Além do propósito da identificação dos componentes saturados e insaturados do biodiesel, o uso da teoria como técnica auxiliar pode contribuir para a identificação de impurezas, tais como: óleo cru (TG) e glicerol (subproduto da reação de transesterificação - método mais utilizado para a produção de biodiese ${ }^{31-33}$ ), ponto importante a ser analisado, já que as propriedades físicas de tais impurezas não são compatíveis com aquelas adequadas para motores do ciclo Diesel. ${ }^{31,33,34}$ A Figura 6 apresenta três espectros de uma mesma composição de EMAG - baseada na composição do biodiesel derivado do óleo de soja - sendo que dois deles mimetizam a contaminação da mistura por triglicerídeos e glicerina. Como se pode perceber pelos espectros, diferenças não são notadas com relação à contaminação do biodiesel por quantidades pequenas de óleo cru. Este resultado já era esperado, uma vez que os espectros de TG e EAG se assemelham muito, haja vista a grande semelhança química.

O resultado que chama maior atenção é aquele derivado da adição de glicerol aos espectros simulados. Como se podem perceber nos espectros, duas bandas de média intensidade derivadas do glicerol possibilitam a avaliação quanto a uma possível contaminação do

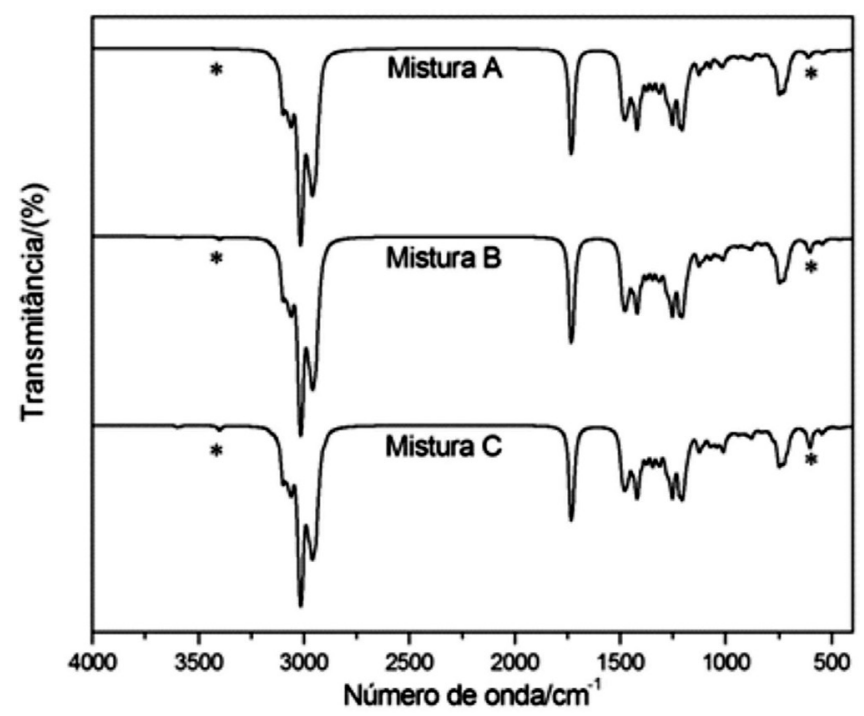

Figura 6. Espectros IV de misturas de EMAG (a) de acordo com as composições: (Mistura A) 16:0 (6\%) + 18:0(4\%) + 18:1 (25\%) + 18:2(50\%) + 18:3 $(5 \%) ;($ Mistura B) 16:0 (6\%) + 18:0 (4\%) + 18:1 (25\%) + 18:2(50\%) + 18:3 $(5 \%)+1 \%$ de cada um dos triglicerídeos com cadeias: 16:0, 18:0, 18:1, 18:2 e 18:3 + 5\% de glicerol e (Mistura C) 16:0 (6\%) + 18:0 (4\%) + 18:1 (25\%) $+18: 2(50 \%)+18: 3(5 \%)+$ glicerol $(10 \%)$. No espectro infravermelho do glicerol (b) é possível observar as principais bandas de distinção 
biodiesel; uma em $3400 \mathrm{~cm}^{-1}$ ( $\mathrm{vOH}$ de álcool primário) e outra em $601 \mathrm{~cm}^{-1}$ ( $\delta \mathrm{OH}$ fora o plano). A primeira, embora seja perceptível, não apresenta nitidez suficiente a ponto de possibilitar uma análise quantitativa. Entretanto, a segunda banda relatada além de apresentar uma grande intensidade, localiza-se em uma região do espectro dos TG e EMAG que não apresenta bandas características destes.

\section{CONCLUSÕES E PERSPECTIVAS}

A interpretação dos espectros infravermelho experimentais em associação com os espectros teóricos conferiu informações auxiliares importantes para a caracterização de EMAG. A distinção entre compostos saturados e insaturados mostrou-se bastante clara e de grande aplicabilidade para a investigação de sistemas reais complexos, que necessitam de um parâmetro confiável para o desenvolvimento de técnicas de análise. Da mesma forma, a detecção de impurezas (glicerol) na mistura de EMAG resultante mostrou-se viável pela análise dos espectros IV. O intento de comparação entre os espectros teóricos e experimentais visa uma possibilidade de análise a baixo custo, com uma reprodutibilidade adequada, partindo de um padrão confiável. No campo da metrologia voltada para biocombustíveis esse é um objetivo iminente. Dessa forma, faz-se necessário que o modelo teórico traduza bem a realidade verificada pelos espectros. No presente estudo foi mostrado que mesmo utilizando um nível de teoria quântico modesto, o perfil espectral é reproduzido de forma satisfatória, permitindo auxiliar o experimentalista na interpretação do espectro com relação à presença de componentes específicos. A partir de estudos exploratórios tal como o aqui apresentado, pode-se vislumbrar a prospecção de um modelo útil de análise pautado em informações teóricas confirmadas por dados experimentais. Nosso grupo continua estudando as características do modelo teórico, visando incluir fatores moleculares relevantes para a representação do sistema real, tal como interações moleculares.

\section{AGRADECIMENTOS}

Este trabalho integra uma parceria entre UFJF, UFMG e INMETRO. Os autores gostariam de agradecer às Agências Brasileiras de Fomento CNPq, CAPES e FAPEMIG. Parte desse projeto é também resultado da colaboração entre pesquisadores da Rede Mineira de Química (RQ-MG), um projeto financiado pela FAPEMIG.

\section{REFERÊNCIAS}

1. Demirbas, A.; Progress in Energy and Combustion Science 2007, 33, 1.

2. Demirbas, A.; Energy Conversion and Management 2008, 49, 2106.

3. Bozbas, K.; Renewable \& Sustainable Energy Reviews 2008, 12, 542.

4. Demirbas, A.; Energy Policy 2007, 35, 4661.

5. Demirbas, A.; Energy Conversion and Management 2008, 49, 125.

6. Sharma, Y. C.; Singh, B.; Upadhyay, S. N.; Fuel 2008, 87, 2355.

7. Saxena, R. C.; Adhikari, D. K.; Goyal, H. B.; Renewable \& Sustainable Energy Reviews 2009, 13, 167.

8. Yang, L.; Zhang, A.; Zheng, X.; Energy Fuels 2009, 23, 3859.

9. Nunes, A. A.; Franca, A. S.; Oliveira, L. S.; Bioresour. Technol. 2010, 100,1786

10. Sims, R. E. H.; Mabee, W.; Saddler, J. N.; Taylor, M.; Bioresour. Technol. 2010, 101, 1570.

11. http://www.mme.gov.br/mme/galerias/arquivos/publicacoes/BEN/2_BEN_-_Ano_Base/1_-_BEN_Portugues_-_Inglxs_-_Completo.pdf, acessada em Agosto 2012.

12. http://www.anp.gov.br/?pg=58071\&m=\&t1=\&t2=\&t3=\&t4=\&ar=\&ps $=\&$ cachebust $=1334831449473$, acessada em Agosto 2012.
13. http://www.faap.br/cees/biodiesel/pdf/ricardo_borges_gomide.pdf, acessada em Agosto 2012.

14. Demirbas, A.; Applied Energy 2009, 86, S108.

15. http://nxt.anp.gov.br/nxt/gateway.d11/leg/folder_portarias_anp/portarias_anp_tec/2003/setembro/panp\%20255\%20-\%202003.xml, acessada em Agosto 2012.

16. http://nxt.anp.gov.br/nxt/gateway.d11/leg/resolucoes_anp/2004/ novembro/ranp\%2042\%20-\%202004.xml, acessada em Agosto 2012.

17. Pinto, A. C.; Guarieiro, L. L. N.; Rezende, M. J. C.; Ribeiro, N. M.; Torres, E. A.; Lopes, W. A.; Pereira, P. A. P.; de Andrade, J. B.; J. Braz. Chem. Soc. 2005, 16, 1313.

18. Demirbas, A.; Energy Conversion and Management 2002, 43, 2349.

19. Mohamadi, F.; Richards, N. G. J.; Guida, W. C.; Liskamp, R.; Lipton, M.; Caufield, C.; Chang, G.; Hendrickson, T.; Still, W. C.; J. Comput. Chem. 1990, 11, 440.

20. Weiner, S. J.; Kollman, P. A.; Case, D. A.; Singh, U. C.; Ghio, C.; Alagona, G.; Profeta Jr., S.; Weiner, P.; J. Am. Chem. Soc. 1984, 106, 765 .

21. Cornell, W. D.; Cieplak, P.; Bayly, C. I.; Gould, I. R.; Merz Jr., K. M.; Ferguson, D. M.; Spellmeyer, D. C.; Fox, T.; Caldwell, J. W.; Kollman, P. A.; J. Am. Chem. Soc. 1995, 117, 5179.

22. Almeida, E. W. C.; Novato, W. T. G.; Anconi, C. P. A.; De Oliveira, M. A. L.; De Almeida, W. B.; Dos Santos, H. F.; J. Inclusion Phenom. Macrocyclic Chem. 2011, 71, 103.

23. Hohemberg, P.; Kohn, W.; Phys. Rev. B: Condens. Matter Mater. Phys 1964, 136, 864; Kohn, W.; Sham, L.; J. Phys. Rev. 1965, 140, A1133; Vosko, S. H.; Wilk, L.; Nusair, M.; Can. J. Phys. 1980, 58, 1200.

24. Frisch, M. J.; Trucks, G. W.; Schlegel, H. B.; Scuseria, G. E.; Robb, M. A.; Cheeseman, J. R.; Montgomery, Jr., J. A.; Vreven, T.; Kudin, K. N.; Burant, J. C.; Millam, J. M.; Iyengar, S. S.; Tomasi, J.; Barone, V.; Mennucci, B.; Cossi, M.; Scalmani, G.; Rega, N.; Petersson, G. A.; Nakatsuji, H.; Hada, M.; Ehara, M.; Toyota, K.; Fukuda, R.; Hasegawa, J.; Ishida, M.; Nakajima, T.; Honda, Y.; Kitao, O.; Nakai, H.; Klene, M.; Li, X.; Knox, J. E.; Hratchian, H. P.; Cross, J. B.; Bakken, V.; Adamo, C.; Jaramillo, J.; Gomperts, R.; Stratmann, R. E.; Yazyev, O.; Austin, A. J.; Cammi, R.; Pomelli, C.; Ochterski, J. W.; Ayala, P. Y.; Morokuma, K.; Voth, G. A.; Salvador, P.; Dannenberg, J. J.; Zakrzewski, V. G.; Dapprich, S.; Daniels, A. D.; Strain, M. C.; Farkas, O.; Malick, D. K.; Rabuck, A. D.; Raghavachari, K.; Foresman, J. B.; Ortiz, J. V.; Cui, Q.; Baboul, A. G.; Clifford, S.; Cioslowski, J.; Stefanov, B. B.; Liu, G.; Liashenko, A.; Piskorz, P.; Komaromi, I.; Martin, R. L.; Fox, D. J.; Keith, T.; Al-Laham, M. A.; Peng, C. Y.; Nanayakkara, A.; Challacombe, M.; Gill, P. M. W.; Johnson, B.; Chen, W.; Wong, M. W.; Gonzalez, C.; Pople, J. A.; Gaussian 03 Revision D.02, Gaussian, Inc., Wallingford CT, 2004.

25. Dos Santos, H. F.; Do Val, A. M. G.; Guimarães, A. C.; De Almeida, W. B.; Quim. Nova 1999, 22, 732.

26. Fan, L.; Ziegler, T.; J. Chem. Phys. 1992, 96, 9005.

27. Pople, J. A.; Scott, A. P.; Wong, M. W.; Radom, L.; Isr. J. Chem. 1993, 33,345 .

28. Scott, A. P.; Radom, L.; J. Phys. Chem. 1996, 100, 16502.

29. Silverstein, R. M.; Bassler, G. C.; Morril, T. C.; Identificação espectrométrica de compostos orgânicos, $3^{\text {a }}$ ed., Guanabara: Rio de Janeiro, 1979.

30. Bringe, N. A. Em Manual de Biodiesel; Knothe, G.; Gerpen, J. V.; Krahl, J.; Ramos, L. P., eds.; Edgard Blücher: São Paulo, 2006, cap. 6.

31. Geris, R.; Dos Santos, N. A. C.; Amaral, B. A.; Maia, I. S.; Castro, V. D.; Carvalho, J. R. M.; Quim. Nova 2007, 30, 1369.

32. Ma, F.; Hanna, M. A.; Bioresour. Technol. 1999, 70, 1.

33. Schuchardt, U.; Sercheli, R.; Vargas, R. M.; J. Braz. Chem. Soc. 1998, 9, 199.

34. Suarez, P. A. Z.; Meneghetti, S. M. P.; Meneghetti, M. R.; Wolf, C. R.; Quim. Nova 2007, 30, 667. 